\title{
On the stochastic 2-D motion of a Bingham fluid
}

\author{
Jong Uhn Kim
}

\begin{abstract}
We establish the existence and uniqueness of a strong solution to the initial boundary value problem associated with the motion of a Bingham fluid in a two-dimensional domain with random noise. We also prove the existence of an invariant measure for a certain class of noise. When the deterministic forcing is sufficiently small and the multiplicative noise is almost linear, it is shown that extinction of a solution occurs in a finite time almost surely.
\end{abstract}

Mathematics Subject Classification (1991). 35Q30, 35Q35, 35R60, 60H15, $60 \mathrm{H} 30$.

Keywords. Bingham fluid, Navier-Stokes equations, Subdifferential, Random noise, Invariant measure, Multiplicative noise,

Extinction of a solution.

\section{Introduction}

In this paper, we will discuss the initial boundary value problem associated with the motion of a Bingham fluid with random noise in a two dimensional bounded domain $G$ with smooth boundary $\partial G$. The problem is formulated as follows.

$$
\begin{aligned}
& \frac{\partial u}{\partial t}-\nu \Delta u+(u \cdot \nabla) u+\nabla p-g \nabla \cdot\left(\frac{D_{i j}(u)}{\sqrt{D_{I I}(u)}}\right)=f+h(u) \frac{d W}{d t} \\
& \quad(t, x) \in(0, T) \times G \\
& \nabla \cdot u=0, \quad(t, x) \in(0, T) \times G \\
& u(t, x)=0, \quad(t, x) \in(0, T) \times \partial G \\
& u(0, x)=u_{0}(x), \quad x \in G
\end{aligned}
$$

where $u$ is the velocity and the right hand side of (0.1) consists of deterministic external force and random noise. Here we set the mass density to be one. Equation (0.1) is a formal expression of the conservation of the linear momentum based on the constituent law: 


$$
\sigma_{i j}=-p \delta_{i j}+g \frac{D_{i j}(u)}{\sqrt{D_{I I}(u)}}+2 \nu D_{i j}(u)
$$

where $\sigma=\left\{\sigma_{i j}\right\}$ is the stress tensor, $p$ is the pressure, $\delta_{i j}$ is the Kronecker delta, $\nu>0$ is the viscosity, $g>0$ is the yield limit, and

$$
\begin{array}{r}
D_{i j}(u)=\frac{1}{2}\left(\frac{\partial u_{i}}{\partial x_{j}}+\frac{\partial u_{j}}{\partial x_{i}}\right), \quad i, j=1,2 \\
D_{I I}(u)=\frac{1}{2} \sum_{i, j=1}^{2}\left(D_{i j}(u)\right)^{2}
\end{array}
$$

The above problem is essentially a hybrid of the stochastic Navier-Stokes equations and a variational inequality. The last term in the left-hand side of (0.1) stands for the subdifferential of the convex functional

$$
J(u)=2 g \int_{G} \sqrt{D_{I I}(u)} d x
$$

and the problem can be described by a variational inequality in the deterministic case $h(\cdot) \equiv 0$; see [8]. This form of variational inequality does not carry over to the stochastic case in a natural way because of Ito's formula. Instead, we will adopt the formulation in the spirit of [13]. For this, we use essentially an equivalent formulation of the deterministic problem established in [8]. In particular, the technical details for handling the singular term in (0.1) can be found in [8, p. 322-326]. The precise definition of a solution to $(0.1)-(0.4)$ will be given in Sect. 1 .

In this work, we will address three topics. First we establish the existence and uniqueness of a solution in the natural function class in a given probability space, which we call a strong solution. Thanks to recent developments in the study of 2D stochastic Navier-Stokes equations, we can substantially simplify technical details. There are some different approaches to the existence of a solution of 2D stochastic Navier-Stokes equations. Among these, the method due to [17] is particularly useful to our problem. This method has been recently generalized in [16] to cover broad class of stochastic locally monotone equations, and we will follow the technical framework of [16]. Stochastic Navier-Stokes equations are discussed as an example in [16], to which readers are referred for various technical details.

We then consider the existence of an invariant measure for a certain class of random noise. For additive noise, the existence of an invariant measure for the 2-D stochastic Navier-Stokes equations was established in [9] using dissipativity property of the stochastic dynamic. Also, see [3] which used the Krylov-Bogoliubov method. These methods can be applied to more general noise under some restrictive conditions, and there are many other works. For an extensive list of references on the invariant measures of the stochastic NavierStokes equations, see [10]. However, for multiplicative noise, there seems to be only a limited number of works which discussed invariant measures; see [11].

Here we consider a class of random noise which includes linear multiplicative noise under some conditions independent of the viscosity $\nu>0$. To 
my knowledge, the existence of an invariant measure under our conditions on random noise has not been discussed even for the 2D stochastic Navier-Stokes equations. One clear distinction between our treatment of the noise and those in earlier works is that we utilize the multiplicative noise to induce a subtle damping.

The uniqueness of an invariant measure for our problem is wide open. The third topic is associated with peculiar properties of Bingham fluids. We will show that extinction of a solution occurs in a finite time almost surely. This is well known for the deterministic case: see [6] and [12]. Under the assumption that the size of deterministic external force is sufficiently small and the random noise is purely multiplicative and almost linear, we will show that solutions become extinct in a finite time almost surely regardless of the size of the initial data. More precisely, it will be shown that the probability that the solution becomes extinct before time $t$ converges to one exponentially fast in large $t$. There are various other physical model equations where solutions become extinct in a finite time; see [5]. One such example is an equation of fast diffusion. For the stochastic equation of fast diffusion, see [1] and [2]. Our result for a Bingham fluid is stronger than those for fast diffusion.

\section{Notation and main results}

We will use the usual notation.

$$
\begin{aligned}
V & =\left\{v \in H_{0}^{1}\left(G ; R^{2}\right) \mid \nabla \cdot v=0 \text { in } \mathrm{G}\right\} \\
\mathcal{H} & =\text { the completion of } V \text { in } L^{2}\left(G ; R^{2}\right) \\
\|v\|_{V} & =\||\nabla v|\|_{L^{2}(G)}, \quad\|v\|_{\mathcal{H}}=\|v\|_{L^{2}\left(G ; R^{2}\right)} \\
V^{*} & =\text { the dual of } V
\end{aligned}
$$

By identifying $\mathcal{H}$ with its dual, we have a Gelfand triple.

$$
V \subset \mathcal{H} \subset V^{*}
$$

We define

$$
\Pi=\text { the projection of } L^{2}\left(G ; R^{2}\right) \text { onto } \mathcal{H}
$$

and let $\hat{\Pi}$ be the mapping: $H^{-1}\left(G ; R^{2}\right) \rightarrow V^{*}$ such that for each $\psi \in$ $H^{-1}\left(G ; R^{2}\right)$,

$$
\langle\hat{\Pi}(\psi), w\rangle_{V^{*}, V}=\langle\psi, w\rangle_{H^{-1}, H_{0}^{1}}, \quad \forall w \in V
$$

where $<\cdot, \cdot>_{H^{-1}, H_{0}^{1}}$ denotes the duality pairing between $H^{-1}\left(G ; R^{2}\right)$ and $H_{0}^{1}\left(G ; R^{2}\right)$. Then, $\hat{\Pi}$ is a continuous linear mapping from $H^{-1}\left(G ; R^{2}\right)$ into $V^{*}$, and

$$
\hat{\Pi}(\psi)=\Pi(\psi) \in \mathcal{H}, \quad \text { if } \psi \in L^{2}\left(G ; R^{2}\right)
$$

So from now on, $\hat{\Pi}$ will be also written as $\Pi$.

Throughout this paper, a stochastic basis $\left(\Omega, \mathcal{F},\left\{\mathcal{F}_{t}\right\}, P\right)$ is given and fixed, where $\left\{\mathcal{F}_{t}\right\}$ is a right continuous filtration over the probability space 
$(\Omega, \mathcal{F}, P)$ such that $\mathcal{F}_{0}$ contains all $P$-negligible subsets of $\mathcal{F} .\left\{B_{k}\right\}_{k=1}^{\infty}$ is a sequence of mutually independent standard Brownian motions on $\left(\Omega, \mathcal{F},\left\{\mathcal{F}_{t}\right\}, P\right)$. Let $T>0$ be given and consider a collection of sets defined by

$\mathcal{G}=\left\{A \in \mathcal{F}_{T} \otimes \mathcal{B}([0, T]) \mid A \cap(\Omega \times[0, t]) \in \mathcal{F}_{t} \otimes \mathcal{B}([0, t])\right.$ for each $\left.\mathrm{t} \in[0, T]\right\}$

Then, $(\Omega \times[0, T], \mathcal{G}, d P \times d t)$ is a finite measure space. Let $\mathcal{X}$ be a separable Banach space. A function $f: \Omega \times[0, T] \rightarrow \mathcal{X}$ is said to be $\mathcal{X}$-valued progressively measurable if $f^{-1}(Q) \in \mathcal{G}$ for each $Q \in \mathcal{B}(\mathcal{X})$, where $\mathcal{B}(\mathcal{X})$ stands for the collection of all Borel subsets of $\mathcal{X}$. For $1 \leq p<\infty, 1 \leq r<\infty$, let $\mathcal{S}_{p, r}$ be the set of equivalent classes of $\mathcal{X}$-valued progressively measurable functions $f$ such that

$$
\int_{\Omega}\left(\int_{0}^{T}\|f\|_{\mathcal{X}}^{r} d t\right)^{\frac{p}{r}} d P<\infty
$$

Next let

$$
\mathcal{Y}_{r}=L^{r}([0, T], \mathcal{B}([0, T]), d t ; \mathcal{X})
$$

Each member of $\mathcal{S}_{p, r}$ belongs to an equivalent class in $L^{p}\left(\Omega, \mathcal{F}, d P ; \mathcal{Y}_{r}\right)$. By the usual convention, we regard an equivalent class in $L^{p}\left(\Omega, \mathcal{F}, d P ; \mathcal{Y}_{r}\right)$ as a member of $\mathcal{S}_{p, r}$ if it contains a representative from $\mathcal{S}_{p, r}$. See [14, p. 131].In this way, it is easy to see that $\mathcal{S}_{p, r}$ is a closed linear subspace of $L^{p}\left(\Omega, \mathcal{F}, d P ; \mathcal{Y}_{r}\right)$. Hence, it is also weakly closed.

We will need the following fact, which can be proved by approximating $L^{\infty}$-norm by $L^{r}$-norm. However, one cannot use directly weak star convergence because $L^{\infty}(0, T ; \mathcal{X})$ may not have the Radon-Nikodym property with respect to the probability measure $P$. In this regard, see [7, p. 98].

Lemma 1.1. Let $\mathcal{X}$ be a separable Hilbert space. Let $\left\{f_{n}\right\}_{n=1}^{\infty}$ be a sequence of $\mathcal{X}$-valued progressively measurable functions such that for some $1<p<\infty$,

$$
\int_{\Omega} \text { ess } \sup _{0 \leq t \leq T}\left\|f_{n}(t)\right\|_{\mathcal{X}}^{p} d P \leq C, \quad \text { for all } n \geq 1 \text { for some constant } C>0
$$

Then, there is an $\mathcal{X}$-valued progressively measurable function $f$ and a subsequence $\left\{f_{n_{k}}\right\}_{k=1}^{\infty}$ such that

$$
\int_{\Omega} e s s \sup _{0 \leq t \leq T}\|f(t)\|_{\mathcal{X}}^{p} d P \leq C
$$

and

$$
f_{n_{k}} \rightarrow f \quad \text { weakly in } L^{p}\left(\Omega, \mathcal{F}, d P ; \mathcal{Y}_{r}\right), \quad \text { for every } 1 \leq r<\infty .
$$

For the general information on stochastic analysis, see $[4,14]$ and [18].

Throughout this paper, we assume that

$$
h(v) d W=\sum_{k=1}^{\infty} h_{k}(v) d B_{k}
$$


where for each $k=1,2, \ldots, h_{k}$ is a mapping from $\mathcal{H}$ into itself such that

$$
\begin{array}{r}
\sum_{k=1}^{\infty}\left\|h_{k}(v)\right\|_{\mathcal{H}}^{2} \leq C_{1}\|v\|_{\mathcal{H}}^{2}+C_{2}, \quad \forall v \in \mathcal{H} \\
\sum_{k=1}^{\infty}\left\|h_{k}(v)-h_{k}(w)\right\|_{\mathcal{H}}^{2} \leq C_{3}\|v-w\|_{\mathcal{H}}^{2}, \quad \forall v, w \in \mathcal{H}
\end{array}
$$

for some nonnegative constants $C_{1}, C_{2}, C_{3}$.

We adopt the following definition of a strong solution of (0.1)-(0.4) based on [8, p. 322].

Definition 1.2. $u$ is called a strong solution on the interval $[0, T]$ of $(0.1)-(0.4)$ if the following conditions are satisfied.

(i) $\mathrm{u}$ is a $\mathrm{V}$-valued progressively measurable process such that

$$
u \in L^{2}(\Omega ; C([0, T] ; \mathcal{H})) \cap L^{2}\left(\Omega ; L^{2}(0, T ; V)\right)
$$

(ii) There is a tensor $\xi=\left\{\xi_{i j}\right\}_{i, j=1,2}$ such that

$$
\xi_{i j} \in L^{\infty}(\Omega \times[0, T] \times G, \mathcal{G} \otimes \mathcal{B}(G), d P \times d t \times d x), i, j=1,2
$$

and, $P$-almost surely,

$$
\begin{gathered}
\xi_{i j}=\xi_{j i}, i, j=1,2, \text { and } \\
\sum_{j=1}^{2} \xi_{j j}=0, \text { in the sense of distributions over }(0, T) \times G \\
\sum_{i, j=1,2}\left(\xi_{i j}\right)^{2} \leq 2, \text { for almost all }(t, x) \in[0, T] \times G \\
\sum_{i, j=1,2} \xi_{i j} D_{i j}(u)=2 \sqrt{D_{I I}(u)}, \text { for almost all }(t, x) \in[0, T] \times G \\
J(v)-J(u) \geq\langle-g \Pi(\nabla \cdot \xi), v-u\rangle_{V^{*}, V}
\end{gathered}
$$

holds for all $v \in V$, for almost all $t \in[0, T]$, where $J(\cdot)$ is defined by $(0.5)$.

(iii) It holds $P$-almost surely that

$$
\begin{aligned}
u(t)= & u_{0}+\nu \int_{0}^{t} \Pi \Delta u d s-\int_{0}^{t} \Pi((u \cdot \nabla) u) d s+g \int_{0}^{t} \Pi(\nabla \cdot \xi) d s+\int_{0}^{t} f d s \\
& +\int_{0}^{t} h(u) d W(s), \quad \text { in } V^{*}
\end{aligned}
$$

for all $t \in[0, T]$, where we identify two $V^{*}$-valued $\mathcal{F}_{T} \otimes \mathcal{B}([0, T])$-measurable stochastic processes if they are equal $d P \times d t$-almost everywhere.

Theorem 1.3. Let $T>0$ be given and assume (1.1)-(1.3). Suppose that $f \in$ $L^{4}\left(0, T ; V^{*}\right), u_{0}$ is $\mathcal{F}_{0}$-measurable, and $u_{0} \in L^{4}(\Omega ; \mathcal{H})$. Then, there is a unique strong solution $u$ of $(0.1)-(0.4)$ such that

$$
u \in L^{4}(\Omega ; C([0, T] ; \mathcal{H})) \cap L^{2}\left(\Omega ; L^{2}(0, T ; V)\right)
$$


For the existence of an invariant measure, we assume that $f$ is independent of time, and

$$
\sum_{k=1}^{\infty}\left|\left\langle h_{k}(v), v\right\rangle_{\mathcal{H}}\right|^{2} \geq C_{4}\|v\|_{\mathcal{H}}^{4}-C_{5}
$$

for some constants $C_{4}>0, C_{5} \geq 0$, such that

$$
2 C_{4}>C_{1}
$$

Theorem 1.4. Suppose that $f$ in (0.1) is independent of time and $f \in V^{*}$. Under the conditions (1.1)-(1.3) and (1.10)-(1.11), there is an invariant measure for (0.1)-(0.3).

Remark 1.5. Suppose that for each $k \geq 1$,

$$
h_{k}(v)=a_{k} v+g_{k}
$$

where $a_{k}$ is a constant and $g_{k} \in \mathcal{H}$ such that

$$
0<\sum_{k=1}^{\infty}\left|a_{k}\right|^{2}<\infty, \quad \sum_{k=1}^{\infty}\left\|g_{k}\right\|_{\mathcal{H}}^{2}<\infty
$$

Then, (1.2)-(1.3) and (1.10)-(1.11) are satisfied. We can take

$$
C_{1}=\frac{5}{4} \sum_{k=1}^{\infty}\left|a_{k}\right|^{2}, \quad C_{3}=\sum_{k=1}^{\infty}\left|a_{k}\right|^{2}, \quad C_{4}=\frac{3}{4} \sum_{k=1}^{\infty}\left|a_{k}\right|^{2}
$$

and choose suitable $C_{2}>0$ and $C_{5}>0$ accordingly.

For finite time extinction of a solution, we need more conditions on the noise terms. We rewrite (1.2) with $C_{2}=0$ and (1.10) with $C_{5}=0$.

$$
\begin{array}{r}
\sum_{k=1}^{\infty}\left\|h_{k}(v)\right\|_{\mathcal{H}}^{2} \leq C_{1}\|v\|_{\mathcal{H}}^{2}, \quad \forall v \in \mathcal{H} \\
\sum_{j=1}^{\infty}\left|\left\langle h_{j}(v), v\right\rangle_{\mathcal{H}}\right|^{2} \geq C_{4}\|v\|_{\mathcal{H}}^{4}
\end{array}
$$

Under the condition (1.11), we choose a number $0<r<\frac{1}{2}$ such that

$$
2(1-r) C_{4}>C_{1}
$$

We need the following version of Korn's inequality proved in [19].

$$
\|w\|_{L^{2}\left(G ; R^{2}\right)} \leq K_{1} \int_{G} \sqrt{D_{I I}(w)} d x, \quad \forall w \in H_{0}^{1}\left(G ; R^{2}\right)
$$

We also need the Poincare's inequality.

$$
\|v\|_{\mathcal{H}} \leq K_{2}\|v\|_{V}, \quad \forall v \in V
$$

Here $K_{1}$ and $K_{2}$ are positive constants depending only on $G$.

We assume that $f \in L^{\infty}(0, \infty ; \mathcal{H})$ and

$$
\|f\|_{L^{\infty}(0, \infty ; \mathcal{H})}<\frac{2 g}{K_{1}}
$$


Let $u$ be a solution of $(0.1)-(0.4)$, and define

$$
\tau=\left\{\begin{array}{l}
\inf \left\{t \geq 0 \mid\|u(t)\|_{\mathcal{H}}=0\right\} \\
\infty, \quad \text { if the set }\{\cdots\} \text { is empty }
\end{array}\right.
$$

Then, $\tau$ is a stopping time.

Theorem 1.6. Let $u_{0}$ be $\mathcal{H}$-valued $\mathcal{F}_{0}$-measurable and $u_{0} \in L^{4}(\Omega ; \mathcal{H})$. Under the conditions (1.3), (1.12)-(1.14), and (1.17), it holds that

$$
u(t)=0, \quad \forall t \geq \tau, \quad \text { P-almost surely, }
$$

and that

$$
P\{\tau<t\} \geq 1-\frac{C}{e^{\lambda t}-1} E\left(\left\|u_{0}\right\|_{\mathcal{H}}^{2 r}\right), \quad \forall t>0
$$

Here, $0<r<\frac{1}{2}$ is a constant satisfying (1.14), and $C$ and $\lambda$ are positive constants independent of $u_{0}$ and $t>0$.

Remark 1.7. Suppose that for each $k \geq 1$,

$$
h_{k}(v)=a_{k} v
$$

where $a_{k}$ is a constant such that

$$
0<\sum_{k=1}^{\infty}\left|a_{k}\right|^{2}<\infty
$$

Then, we can take

$$
C_{1}=C_{3}=C_{4}=\sum_{k=1}^{\infty}\left|a_{k}\right|^{2}
$$

to satisfy (1.11)-(1.13).

\section{Proof of Theorem 1.3}

The proof consists of two parts. In the first part, we regularize $\sqrt{D_{I I}(u)}$ in (0.1) by $\sqrt{\epsilon+D_{I I}(u)}, \epsilon>0$. For the existence of a solution to the regularized problem, we can directly borrow the existence result from [16]. For this, it is enough to check some basic properties of the regularized term. In the second part, we will obtain a solution of (0.1)-(0.4) as a limit of a suitable sequence of solutions of the regularized problem as the parameter $\epsilon \rightarrow 0$. We will present all the technical details.

Part 1. We consider a regularized problem.

$$
\begin{aligned}
u_{\epsilon}(t)= & u_{0}+\nu \int_{0}^{t} \Pi \Delta u_{\epsilon} d s-\int_{0}^{t} \Pi\left(\left(u_{\epsilon} \cdot \nabla\right) u_{\epsilon}\right) d s \\
& +g \int_{0}^{t} \Pi\left(\nabla \cdot \xi_{\epsilon}\left(u_{\epsilon}\right)\right) d s+\int_{0}^{t} f d s+\int_{0}^{t} h\left(u_{\epsilon}\right) d W(s), \quad \text { in } V^{*}, \quad \forall t \in[0, T]
\end{aligned}
$$


where for each $\epsilon>0, \xi_{\epsilon}(\cdot)=\left\{\xi_{\epsilon, i j}(\cdot)\right\}_{i, j=1,2}$ is defined by

$$
\xi_{\epsilon, i j}(v)=\frac{D_{i j}(v)}{\sqrt{\epsilon+D_{I I}(v)}}, \quad i, j=1,2, \quad v \in V
$$

As in (1.9), we identify two $V^{*}$-valued $\mathcal{F}_{T} \otimes \mathcal{B}([0, T])$-measurable stochastic processes if they are equal $d P \times d t$-almost everywhere.

Let us consider the convex functional with domain $V$

$$
J_{\epsilon}(v)=2 g \int_{G} \sqrt{\epsilon+D_{I I}(v)} d x, \quad \forall v \in V
$$

Then, it is easy to see that $J_{\epsilon}(\cdot)$ is Gâteaux differentiable, and its derivative $\partial J_{\epsilon}(\cdot)$ is given by

$$
\begin{aligned}
<\partial J_{\epsilon}(v), w>_{V^{*}, V} & =g \sum_{i, j=1,2} \int_{G} \frac{D_{i j}(v) D_{i j}(w)}{\sqrt{\epsilon+D_{I I}(v)}} d x \\
& =-g\left\langle\Pi\left(\nabla \cdot \xi_{\epsilon}(v)\right), w\right\rangle_{V^{*}, V}
\end{aligned}
$$

It follows that

$$
\begin{aligned}
&-\left\langle\Pi\left(\nabla \cdot \xi_{\epsilon}(v)\right)-\Pi\left(\nabla \cdot \xi_{\epsilon}(w)\right), v-w\right\rangle_{V^{*}, V} \geq 0, \quad \forall v, w \in V \quad \\
&\left\|\Pi\left(\nabla \cdot \xi_{\epsilon}(v)\right)\right\|_{V^{*}}=\sup _{\|w\|_{V} \leq 1}\left\langle\Pi\left(\nabla \cdot \xi_{\epsilon}(v)\right), w\right\rangle_{V^{*}, V} \\
&=\sup _{\|w\|_{V} \leq 1}\left|\sum_{i, j=1,2} \int_{G} \frac{D_{i j}(v) D_{i j}(w)}{\sqrt{\epsilon+D_{I I}(v)}} d x\right| \\
& \leq \sup _{\|w\|_{V} \leq 1} \sqrt{2} \sum_{i, j=1,2} \int_{G}\left|D_{i j}(w)\right| d x \leq C, \quad \forall v \in V, \quad \forall \epsilon>0,
\end{aligned}
$$

for some positive constant $C$, and

$$
0 \leq J_{\epsilon}(v)+g\left\langle\Pi\left(\nabla \cdot \xi_{\epsilon}(v)\right), v\right\rangle_{V^{*}, V} \leq 2 g \sqrt{\epsilon} \int_{G} d x, \quad \forall v \in V
$$

It is also easy to see that for each $v, w, y \in V$,

$$
\left\langle\Pi\left(\nabla \cdot \xi_{\epsilon}(v+\eta y)\right), w\right\rangle_{V^{*}, V} \rightarrow\left\langle\Pi\left(\nabla \cdot \xi_{\epsilon}(v)\right), w\right\rangle_{V^{*}, V}
$$

as $\eta \rightarrow 0$.

By virtue of (2.4)-(2.6) and (2.8), we can incorporate $\Pi\left(\nabla \cdot \xi_{\epsilon}\left(u_{\epsilon}\right)\right)$ with other terms in (2.1) to satisfy all the required conditions in Theorem 1.1 of [16], which yields the following result.

Lemma 2.1. Let $T>0$ be given, and $f \in L^{4}\left(0, T ; V^{*}\right)$. Suppose that $u_{0}$ is $\mathcal{H}$-valued $\mathcal{F}_{0}$-measurable and belongs to $L^{4}(\Omega ; \mathcal{H})$. Then, for each $\epsilon>0$, there is a unique solution $u_{\epsilon}$ of (2.1) such that $u_{\epsilon}$ is $V$-valued progressively measurable, and

$$
u_{\epsilon} \in L^{4}(\Omega ; C([0, T] ; \mathcal{H})) \cap L^{2}\left(\Omega ; L^{2}(0, T ; V)\right)
$$


Furthermore, it holds that

$$
E\left(\left\|u_{\epsilon}\right\|_{C([0, T] ; \mathcal{H})}^{4}\right)+E\left(\left\|u_{\epsilon}\right\|_{L^{2}(0, T ; V)}^{2}\right) \leq C_{T} E\left(\left\|u_{0}\right\|_{\mathcal{H}}^{4}\right)+C_{T}
$$

and

$$
E\left(\int_{0}^{T}\left\|u_{\epsilon}(t)\right\|_{L^{4}\left(G ; R^{2}\right)}^{4} d t\right) \leq C_{T} E\left(\left\|u_{0}\right\|_{\mathcal{H}}^{4}\right)+C_{T}
$$

where $C_{T}$ denotes positive constants independent of $g, \epsilon$ and $u_{0}$.

\section{Part 2}

We will construct a solution of (0.1)-(0.4) according to Definition of 1.2. Let us write

$$
A(t, v)=\nu \Pi \Delta v-\Pi((v \cdot \nabla) v)+f(t)
$$

It follows from (1.3) and the well-known inequalities associated with the map

$$
v \mapsto \Pi((v \cdot \nabla) v)
$$

that

$$
\begin{aligned}
& 2\langle A(t, v)-A(t, w), v-w\rangle_{V^{*}, V}+\sum_{k=1}^{\infty}\left\|h_{k}(v)-h_{k}(w)\right\|_{\mathcal{H}}^{2} \\
& \quad \leq\left(\kappa_{1}+\kappa_{2}\|w\|_{L^{4}\left(G ; R^{2}\right)}^{4}\right)\|v-w\|_{\mathcal{H}}^{2}, \quad \forall v, w \in V, \quad \forall t \in[0, T]
\end{aligned}
$$

for some positive constants $\kappa_{1}, \kappa_{2}$. For the proof of (2.11), see p. 2918 of [16].

We define a function class

$$
\begin{aligned}
\mathcal{K}= & \{\phi \mid \phi \text { is } \mathrm{V} \text {-valued progressively measurable such that } \\
& \left.\phi \in L^{2}(\Omega \times[0, T] ; V) \cap L^{4}\left(\Omega \times[0, T] ; L^{4}\left(G ; R^{2}\right)\right)\right\}
\end{aligned}
$$

Choose any $\phi \in \mathcal{K}$ and define

$$
\Phi(t)=\int_{0}^{t}\left(\kappa_{1}+\kappa_{2}\|\phi(s)\|_{L^{4}\left(G ; R^{2}\right)}^{4}\right) d s, \quad t \in[0, T]
$$

We note that $\Phi(T)<\infty, P$-almost surely, and that if $q$ is a nonnegative scalar function which belongs to $L^{\infty}(0, T)$,

$$
\int_{0}^{T} e^{-\Phi(t)} \Phi^{\prime}(t) q(t) d t \leq\|q\|_{L^{\infty}(0, T)}\left(1-e^{-\Phi(T)}\right) \leq\|q\|_{L^{\infty}(0, T)}
$$

Thus, by (2.9),

$$
E\left(\int_{0}^{T} e^{-\Phi(t)}\left(\kappa_{1}+\kappa_{2}\|\phi(t)\|_{L^{4}\left(G ; R^{2}\right)}^{4}\right)\left\|u_{\epsilon}(t)\right\|_{\mathcal{H}}^{2} d t\right) \leq C
$$

for some constant $C>0$ independent of $\epsilon>0$ and $\phi$. 
By Ito's formula, we derive from (2.1)

$$
\begin{aligned}
& E\left(e^{-\Phi(t)}\left\|u_{\epsilon}(t)\right\|_{\mathcal{H}}^{2}\right)-E\left(\left\|u_{0}\right\|_{\mathcal{H}}^{2}\right)=E\left(\int _ { 0 } ^ { t } e ^ { - \Phi ( s ) } \left[2\left\langle A\left(s, u_{\epsilon}(s)\right), u_{\epsilon}(s)\right\rangle_{V^{*}, V}\right.\right. \\
& \left.\left.\quad+\sum_{k=1}^{\infty}\left\|h_{k}\left(u_{\epsilon}(s)\right)\right\|_{\mathcal{H}}^{2}-\left(\kappa_{1}+\kappa_{2}\|\phi(s)\|_{L^{4}\left(G ; R^{2}\right)}^{4}\right)\left\|u_{\epsilon}(s)\right\|_{\mathcal{H}}^{2}\right] d s\right) \\
& \quad+E\left(\int_{0}^{t} e^{-\Phi(s)} 2 g\left\langle\Pi\left(\nabla \cdot \xi_{\epsilon}\left(u_{\epsilon}(s)\right)\right), u_{\epsilon}(s)\right\rangle_{V^{*}, V} d s\right), \quad \forall t \in[0, T] .(2.13)
\end{aligned}
$$

By (2.9)-(2.10), we have

$$
E\left(\int_{0}^{T}\left\|A\left(t, u_{\epsilon}(t)\right)\right\|_{V^{*}}^{2} d t\right) \leq C
$$

and, by (1.2) and (2.9),

$$
E\left(\sum_{k=1}^{\infty} \int_{0}^{T}\left\|h_{k}\left(u_{\epsilon}(t)\right)\right\|_{\mathcal{H}}^{2} d t\right) \leq C
$$

for some constants $C>0$ independent of $\epsilon>0$. Thus, we can extract a subsequence $\left\{u_{\epsilon_{n}}\right\}_{n=1}^{\infty}$ such that

$$
A\left(\cdot, u_{\epsilon_{n}}(\cdot)\right) \rightarrow \Xi
$$

weakly in $L^{2}\left(\Omega ; L^{2}\left(0, T ; V^{*}\right)\right)$, where $\Xi$ is $V^{*}$-valued progressively measurable,

$$
h_{k}\left(u_{\epsilon_{n}}\right) \rightarrow \theta_{k}
$$

weakly in $L^{2}\left(\Omega ; L^{2}(0, T ; \mathcal{H})\right)$, for each $k \geq 1$, where $\theta_{k}$ is $\mathcal{H}$-valued progressively measurable,

$$
u_{\epsilon_{n}} \rightarrow \hat{u}
$$

weakly in $L^{2}\left(\Omega ; L^{2}(0, T ; V)\right)$, where $\hat{u}$ is $V$-valued progressively measurable. By Lemma 1.1 and (2.9),

$$
E\left(\text { ess } \sup _{t \in[0, T]}\|\hat{u}(t)\|_{\mathcal{H}}^{4}\right)+E\left(\|\hat{u}\|_{L^{2}(0, T ; V)}^{2}\right)<\infty
$$

Next we will extract a subsequence $\xi_{\epsilon_{n}, i j}$ which converges in a suitable manner. Since the mapping

$$
v \mapsto \xi_{\epsilon, i j}(v)=\frac{D_{i j}(v)}{\sqrt{\epsilon+D_{I I}(v)}}, i, j=1,2, \quad \epsilon>0
$$

is continuous from $V$ into $L^{2}(G)$, it follows from the properties of $u_{\epsilon}$ stated in Lemma 2.1 that $\xi_{\epsilon, i j}\left(u_{\epsilon}\right)$ is $L^{2}(G)$-valued progressively measurable, and belongs to an equivalent class in $L^{2}\left(\Omega \times[0, T], \mathcal{G}, d P \times d t ; L^{2}(G)\right)$. Now fix $\epsilon>0$ and $i, j=1,2$. There is a sequence of $L^{2}(G)$-valued $\mathcal{G}$-measurable simple functions $\left\{s_{n}\right\}$ such that $s_{n}$ converges to $\xi_{\epsilon, i j}\left(u_{\epsilon}\right)$, in $L^{2}(\Omega \times[0, T], \mathcal{G}, d P \times$ $\left.d t ; L^{2}(G)\right)$, as $n \rightarrow \infty$. By virtue of the structure of these simple functions, each $s_{n}$ is $\mathcal{G} \otimes \mathcal{B}(G)$-measurable, and $\left\{s_{n}\right\}$ is a Cauchy sequence in $L^{2}(\Omega \times$ 
$[0, T] \times G, \mathcal{G} \otimes \mathcal{B}(G), d P \times d t \times d x)$. Hence, $\xi_{\epsilon, i j}\left(u_{\epsilon}\right)$ belongs to an equivalent class in $L^{2}(\Omega \times[0, T] \times G, \mathcal{G} \otimes \mathcal{B}(G), d P \times d t \times d x)$. It is obvious that

$$
\sum_{i, j=1,2}\left|\xi_{\epsilon, i j}\left(u_{\epsilon}\right)\right|^{2} \leq 2
$$

for $d P \times d t \times d x$-almost all $(\omega, t, x)$. We now consider the sequence $\left\{\xi_{\epsilon_{n}, i j}\left(u_{\epsilon_{n}}\right)\right\}$ where $\left\{u_{\epsilon_{n}}\right\}$ is the sequence in (2.14)-(2.16). We can extract a subsequence still denoted by $\left\{\xi_{\epsilon_{n}, i j}\left(u_{\epsilon_{n}}\right)\right\}$ such that

$$
\begin{array}{lr}
\xi_{\epsilon_{n}, i j}\left(u_{\epsilon_{n}}\right) \rightarrow \xi_{i j}, & \text { weak star in } L^{\infty}(\Omega \times[0, T] \times G, \mathcal{G} \otimes \mathcal{B}(G), d P \times d t \times d x) \\
\xi_{\epsilon_{n}, i j}\left(u_{\epsilon_{n}}\right) \rightarrow \xi_{i j}, & \text { weakly in } L^{2}\left(\Omega \times[0, T], \mathcal{G}, d P \times d t ; L^{2}(G)\right)
\end{array}
$$

and

$$
\sum_{i, j=1,2}\left|\xi_{i j}\right|^{2} \leq 2
$$

for $d P \times d t \times d x$-almost all $(\omega, t, x)$. We can further extract a subsequence still denoted by $\left\{u_{\epsilon_{n}}\right\}$ from the sequence in $(2.14)-(2.16)$ so that $u_{\epsilon_{n}}$ is associated with $\xi_{\epsilon_{n}, i j}\left(u_{\epsilon_{n}}\right)$ by $(2.2)$.

Let us set

$$
u(t)=u_{0}+\int_{0}^{t} \Xi(s) d s+\int_{0}^{t} g \Pi(\nabla \cdot \xi(s)) d s+\sum_{k=1}^{\infty} \int_{0}^{t} \theta_{k}(s) d B_{k}(s), \quad t \in[0, T]
$$

Then, $u$ is $V^{*}$-valued progressively measurable, and by virtue of (2.1), (2.14)(2.16) and (2.20), $u=\hat{u}$ in $V^{*}$ for $d P \times d t$-almost all $(\omega, t) \in \Omega \times[0, T]$. Since $\hat{u}$ is $V$-valued progressively measurable, we see that $u=\hat{u}$ in $V$ for $d P \times d t$ almost all $(\omega, t) \in \Omega \times[0, T]$. By (2.17), we can apply a result due to [15] to conclude that $u \in C([0, T] ; \mathcal{H}), P$-almost surely, and

$$
\begin{gathered}
E\left(\|u\|_{C([0, T] ; \mathcal{H})}^{4}\right)+E\left(\|u\|_{L^{2}(0, T ; V)}^{2}\right)<\infty \\
E\left(e^{-\Phi(t)}\|u(t)\|_{\mathcal{H}}^{2}\right)-E\left(\left\|u_{0}\right\|_{\mathcal{H}}^{2}\right)=E\left(\int _ { 0 } ^ { t } e ^ { - \Phi ( s ) } \left[2\langle\Xi(s), u\rangle_{V^{*}, V}\right.\right. \\
\left.\left.\left.+\sum_{k=1}^{\infty} \| \theta_{k}(s)\right)\left\|_{\mathcal{H}}^{2}-\left(\kappa_{1}+\kappa_{2}\|\phi(s)\|_{L^{4}\left(G ; R^{2}\right)}^{4}\right)\right\| u(s) \|_{\mathcal{H}}^{2}\right] d s\right) \\
+E\left(\int_{0}^{t} e^{-\Phi(s)} 2 g\langle\Pi(\nabla \cdot \xi(s)), u(s)\rangle_{V^{*}, V} d s\right), \quad \forall t \in[0, T]
\end{gathered}
$$


for each $\phi \in \mathcal{K}$, where $\Phi(t)$ is defined by (2.12). With help of (2.11) and (2.14)-(2.16), we can manipulate (2.13) and (2.24) as in [16] to arrive at

$$
\begin{aligned}
& E\left(\int _ { 0 } ^ { T } \psi ( t ) \left[\int _ { 0 } ^ { t } e ^ { - \Phi ( s ) } \left(2\langle\Xi(s)-A(s, \phi(s)), u(s)-\phi(s)\rangle_{V^{*}, V}\right.\right.\right. \\
& \left.\left.\left.\quad+\sum_{k=1}^{\infty}\left(\left\|h_{k}(\phi(s))-\theta_{k}(s)\right\|_{\mathcal{H}}^{2}\right)-\left(\kappa_{1}+\kappa_{2}\|\phi(s)\|_{L^{4}\left(G ; R^{2}\right)}^{4}\right)\|u(s)-\phi(s)\|_{\mathcal{H}}^{2}\right) d s\right] d t\right) \\
& \quad+\underline{\lim }_{\epsilon \rightarrow 0} E\left(\int _ { 0 } ^ { T } \psi ( t ) \left[\int_{0}^{t} e^{-\Phi(s)}\left\langle-2 g \Pi\left(\nabla \cdot \xi_{\epsilon}\left(u_{\epsilon}(s)\right)\right), u_{\epsilon}(s)\right\rangle_{V^{*}, V} d s\right.\right. \\
& \left.\left.\quad+\int_{0}^{t} e^{-\Phi(s)} 2 g\langle\Pi(\nabla \cdot \xi), u(s)\rangle_{V^{*}, V} d s\right] d t\right) \leq 0
\end{aligned}
$$

for every nonnegative $\psi \in L^{\infty}(0, T)$.

In the meantime, it holds $d P \times d t$-almost everywhere that

$$
0 \leq J_{\epsilon}\left(u_{\epsilon}\right)+g\left\langle\Pi\left(\nabla \cdot \xi_{\epsilon}\left(u_{\epsilon}\right)\right), u_{\epsilon}\right\rangle_{V^{*}, V} \leq 2 g \sqrt{\epsilon} \int_{G} d x
$$

and thus, by (2.16),

$$
\begin{aligned}
& \varliminf_{\epsilon_{n} \rightarrow 0} E\left(\chi_{Q} \int_{0}^{T} \rho(t)\left\langle-g \Pi\left(\nabla \cdot \xi_{\epsilon_{n}}\left(u_{\epsilon_{n}}\right)\right), u_{\epsilon_{n}}\right\rangle_{V^{*}, V} d t\right) \\
& =\underset{\lim _{n} \rightarrow 0}{ } E\left(\chi_{Q} \int_{0}^{T} \rho(t) J_{\epsilon_{n}}\left(u_{\epsilon_{n}}\right) d t\right)
\end{aligned}
$$

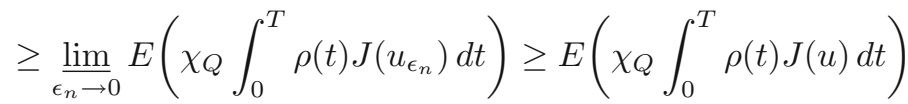

for all nonnegative $\rho \in L^{\infty}(0, T)$ and all $Q \in \mathcal{F}_{T}$. Here $\chi_{Q}$ denotes the characteristic function of the set $Q$.

It follows that

$$
\begin{aligned}
& \underset{\lim _{\epsilon_{n} \rightarrow 0}}{ } E\left(\int_{0}^{T} \psi(t)\left[\int_{0}^{t} e^{-\Phi(s)}\left\langle-2 g \Pi\left(\nabla \cdot \xi_{\epsilon_{n}}\left(u_{\epsilon_{n}}(s)\right)\right), u_{\epsilon_{n}}(s)\right\rangle_{V^{*}, V} d s\right] d t\right) \\
& \quad \geq \int_{0}^{T} \psi(t) \underset{\lim _{n \rightarrow 0}}{ } E\left(\int_{0}^{t} e^{-\Phi(s)}\left\langle-2 g \Pi\left(\nabla \cdot \xi_{\epsilon_{n}}\left(u_{\epsilon_{n}}(s)\right)\right), u_{\epsilon_{n}}(s)\right\rangle_{V^{*}, V} d s\right) d t \\
& \quad \geq 2 \int_{0}^{T} \psi(t) E\left(\int_{0}^{t} e^{-\Phi(s)} J(u) d s\right) d t=2 E\left(\int_{0}^{T}\left[\psi(t) \int_{0}^{t} e^{-\Phi(s)} J(u) d s\right] d t\right)
\end{aligned}
$$

On the other hand,

$$
\begin{aligned}
& E\left(\chi_{Q} \int_{0}^{T} \rho(t)\left[J_{\epsilon}(v)-J_{\epsilon}\left(u_{\epsilon}\right)\right] d t\right) \\
& \quad \geq E\left(\chi_{Q} \int_{0}^{T} \rho(t)\left\langle-g \Pi\left(\nabla \cdot \xi_{\epsilon}\left(u_{\epsilon}\right)\right), v-u_{\epsilon}\right\rangle_{V^{*}, V} d t\right)
\end{aligned}
$$

for all $\epsilon>0$, and all $v \in L^{2}\left(\Omega ; L^{2}(0, T ; V)\right)$, and, by (2.20) and (2.26),

$$
E\left(\chi_{Q} \int_{0}^{T} \rho(t) J(v) d t\right) \geq E\left(\chi_{Q} \int_{0}^{T} \rho(t)\langle-g \Pi(\nabla \cdot \xi), v\rangle_{V^{*}, V} d t\right)
$$


Taking $v=u$, we have

$$
E\left(\chi_{Q} \int_{0}^{T} \rho(t) J(u) d t\right) \geq E\left(\chi_{Q} \int_{0}^{T} \rho(t)\langle-g \Pi(\nabla \cdot \xi), u\rangle_{V^{*}, V} d t\right)
$$

Since (2.31) holds for all $Q \in \mathcal{F}_{T}$ and all nonnegative $\rho \in L^{\infty}(0, T)$,

$$
J(u)+\langle g \Pi(\nabla \cdot \xi), u\rangle_{V^{*}, V} \geq 0
$$

holds for $d P \times d t$-almost all $(\omega, t) \in \Omega \times[0, T]$. We set $\phi=u$ in $(2.25)$, and use (2.28) and (2.32) to arrive at

$$
h_{k}(u)=\theta_{k}, \quad \forall k
$$

and

$$
J(u)=\langle-g \Pi(\nabla \cdot \xi), u\rangle_{V^{*}, V}
$$

for $d P \times d t$-almost all $(\omega, t) \in \Omega \times[0, T]$. By means of $(2.21)$, it holds $d P \times$ $d t$-almost everywhere that

$$
\begin{aligned}
& \langle-g \Pi(\nabla \cdot \xi), u\rangle_{V^{*}, V}=g \int_{G} \sum_{i, j=1,2} \xi_{i j} D_{i j}(u) d x \\
& \quad \leq g \int_{G}\left(\sum_{i, j=1,2}\left|\xi_{i j}\right|^{2}\right)^{\frac{1}{2}} \sqrt{2 D_{I I}(u)} d x \leq 2 g \int_{G} \sqrt{D_{I I}(u)} d x=J(u)
\end{aligned}
$$

Consequently, it holds $P$-almost surely that

$$
\sum_{i, j=1,2} \xi_{i j} D_{i j}(u)=2 \sqrt{D_{I I}(u)}, \quad \text { for almost all }(t, x) \in[0, T] \times G .
$$

By means of $(2.28),(2.33)$ and $(2.34)$, we can reduce (2.25) to

$$
\begin{aligned}
& E\left(\int _ { 0 } ^ { T } \psi ( t ) \left[\int _ { 0 } ^ { t } e ^ { - \Phi ( s ) } \left(2\langle\Xi(s)-A(s, \phi(s)), u(s)-\phi(s)\rangle_{V^{*}, V}\right.\right.\right. \\
& \left.\left.\left.\quad+\sum_{k=1}^{\infty}\left(\left\|h_{k}(\phi(s))-h_{k}(u(s))\right\|_{\mathcal{H}}^{2}\right)-\left(\kappa_{1}+\kappa_{2}\|\phi(s)\|_{L^{4}\left(G ; R^{2}\right)}^{4}\right)\|u(s)-\phi(s)\|_{\mathcal{H}}^{2}\right) d s\right] d t\right) \\
& \quad \leq 0
\end{aligned}
$$

Now we choose any

$$
z \in L^{4}(\Omega \times[0, T], \mathcal{G}, d P \times d t ; V)
$$

and a positive constant $\eta$. Then, $u+\eta z \in \mathcal{K}$, and we may use $\phi=u+\eta z$ in (2.36). After this substitution, we divide both sides by $\eta$ and pass $\eta \rightarrow 0$. It is easy to see that

$$
\begin{aligned}
& E\left(\int_{0}^{T} \psi(t)\left[\int_{0}^{t} e^{-\Psi_{\eta}(s)}\langle A(s, u(s)+\eta z(s)), z(s)\rangle_{V^{*}, V} d s\right] d t\right) \\
& \quad \rightarrow E\left(\int_{0}^{T} \psi(t)\left[\int_{0}^{t} e^{-\Psi(s)}\langle A(s, u(s)), z(s)\rangle_{V^{*}, V} d s\right] d t\right)
\end{aligned}
$$


as $\eta \rightarrow 0$ where

$$
\Psi_{\eta}(t)=\int_{0}^{t}\left(\kappa_{1}+\kappa_{2}\|u(s)+\eta z(s)\|_{L^{4}\left(G ; R^{2}\right)}^{4}\right) d s, \quad t \in[0, T]
$$

and

$$
\Psi(t)=\int_{0}^{t}\left(\kappa_{1}+\kappa_{2}\|u(s)\|_{L^{4}\left(G ; R^{2}\right)}^{4}\right) d s, \quad t \in[0, T]
$$

It follows that

$$
E\left(\int_{0}^{T} \psi(t)\left[\int_{0}^{t} e^{-\Psi(s)}\langle\Xi(s)-A(s, u(s)), z(s)\rangle_{V^{*}, V} d s\right] d t\right) \geq 0
$$

for every $z \in L^{4}(\Omega \times[0, T], \mathcal{G}, d P \times d t ; V)$ and every nonnegative $\psi \in L^{\infty}(0, T)$. Since

$$
e^{-\Psi(\cdot)}(\Xi(\cdot)-A(\cdot, u(\cdot))) \in L^{2}\left(\Omega \times[0, T], \mathcal{G}, d P \times d t ; V^{*}\right)
$$

and $L^{2}\left(\Omega \times[0, T], \mathcal{G}, d P \times d t ; V^{*}\right)$ is embedded into $L^{\frac{4}{3}}(\Omega \times[0, T], \mathcal{G}, d P \times$ $\left.d t ; V^{*}\right)$, we infer from $(2.37)$ that

$$
A(t, u(t))=\Xi(t) \quad \text { in } V^{*}, \quad \text { for } d P \times d t-\operatorname{almost} \text { all }(\omega, t) \in \Omega \times[0, T]
$$

By (2.19), (2.21), (2.30), (2.34) and (2.35), $\xi=\left\{\xi_{i j}\right\}_{i, j=1,2}$ satisfies (1.4), (1.6)-(1.8). Since it holds $P$-almost surely that

$$
\xi_{\epsilon, i j}\left(u_{\epsilon}\right)=\xi_{\epsilon, j i}\left(u_{\epsilon}\right), \quad i, j=1,2,
$$

and

$$
\sum_{j=1}^{2} \xi_{\epsilon, j j}\left(u_{\epsilon}\right)=0 \text {, in the sense of distributions over }(0, T) \times G
$$

it follows from (2.20) that $\xi=\left\{\xi_{i j}\right\}_{i, j=1,2}$ satisfies (1.5). Consequently, it follows from $(2.22),(2.23),(2.33)$, and (2.38) that $u$ is a solution of $(0.1)-(0.4)$ according to Definition 1.2.

Next let $u_{1}$ and $u_{2}$ be solutions with initial data $u_{0,1}$ and $u_{0,2}$, respectively. We write

$$
\Psi_{1}(t)=\int_{0}^{t}\left(\kappa_{1}+\kappa_{2}\left\|u_{1}(s)\right\|_{L^{4}\left(G ; R^{2}\right)}^{4}\right) d s, \quad t \in[0, T]
$$

Let $\xi_{1}$ and $\xi_{2}$ correspond to $u_{1}$ and $u_{2}$, respectively. Then, by virtue of (1.8), it holds $P$-almost surely that

$$
\left\langle\Pi\left(\nabla \cdot \xi_{1}\right)-\Pi\left(\nabla \cdot \xi_{2}\right), u_{1}-u_{2}\right\rangle_{V^{*}, V} \leq 0
$$

for almost all $t \in[0, T]$. Hence, by (2.11) and Ito's formula, we find that

$$
E\left(e^{-\Psi_{1}(t)}\left\|u_{1}(t)-u_{2}(t)\right\|_{\mathcal{H}}^{2}\right) \leq E\left(\left\|u_{0,1}-u_{0,2}\right\|_{\mathcal{H}}^{2}\right), \quad \forall t \in[0, T]
$$

This yields the uniqueness of a solution, and the proof of Theorem 1.3 is complete. 
For later use, we note that (2.10) yields

$$
E\left(\int_{0}^{T}\|u\|_{L^{4}\left(G ; R^{2}\right)}^{4} d t\right) \leq C_{T} E\left(\left\|u_{0}\right\|_{\mathcal{H}}^{4}\right)+C_{T}
$$

\section{Proof of Theorem 1.4}

Suppose that $f$ in (0.1) is independent of time, $f \in V^{*}$, and the conditions (1.1)-(1.3), (1.10) and (1.11) are satisfied. Since $T>0$ is arbitrary in Theorem 1.3 , the unique solution exists on the interval $[0, \infty), P$-almost surely. We first show that the solution is an $\mathcal{H}$-valued Markov process. We need the following lemmas.

Lemma 3.1. Let $0 \leq s<\infty$. Suppose that $\left\{h_{n}\right\}$ is a sequence of $\mathcal{F}_{s}$-measurable functions in $L^{4}(\Omega ; \mathcal{H})$ such that

$$
h_{n} \rightarrow h \quad \text { in } L^{4}(\Omega ; \mathcal{H})
$$

Let $Y(\cdot, s ; h)$ denote the solution of $(0.1)-(0.3)$ on the interval $[s, \infty)$ with initial condition

$$
Y(s, s ; h)=h
$$

Then, for any $s \leq t^{*}<\infty$,

$$
\left\|Y\left(t^{*}, s ; h_{n}\right)-Y\left(t^{*}, s ; h\right)\right\|_{\mathcal{H}} \rightarrow 0 \quad \text { in P-measure }
$$

Proof. Fix $0 \leq s<\infty$. Using $\kappa_{1}, \kappa_{2}$ in (2.11), we define a stopping time for each positive integer $N$,

$$
T_{N}=\inf \left\{t \geq s \mid \int_{s}^{t}\left(\kappa_{1}+\kappa_{2}\|Y(\eta, s ; h)\|_{L^{4}\left(G ; R^{2}\right)}^{4}\right) d \eta \geq N\right\}
$$

We set $T_{N}=\infty$ if the set $\{\cdots\}$ is empty.

We write

$$
\Psi_{s}(t)=\int_{s}^{t}\left(\kappa_{1}+\kappa_{2}\|Y(\eta, s ; h)\|_{L^{4}\left(G ; R^{2}\right)}^{4}\right) d \eta
$$

and

$$
\Theta_{n}(t)=Y\left(t, s ; h_{n}\right)-Y(t, s ; h), \quad t \geq s
$$

Since (2.39) is valid with the interval $[0, t]$ replaced by $\left[s, t \wedge T_{N}\right]$, it holds that

$$
E\left(e^{-\Psi_{s}\left(t \wedge T_{N}\right)}\left\|\Theta_{n}\left(t \wedge T_{N}\right)\right\|_{\mathcal{H}}^{2}\right) \leq E\left(\left\|h_{n}-h\right\|_{\mathcal{H}}^{2}\right), \quad \forall t \geq s .
$$

Choose any $t^{*}>s$. Then,

$$
\left\{T_{N} \leq t^{*}\right\} \subset\left\{\Psi_{s}\left(t^{*}\right) \geq N\right\}
$$

and, by (2.40),

$$
P\left\{\Psi_{s}\left(t^{*}\right) \geq N\right\} \leq \frac{C\left(t^{*}\right)}{N}
$$

where $C\left(t^{*}\right)$ denotes a positive constant independent of $N$ and $n$. Let

$$
\Omega_{N}=\Omega \backslash\left\{\Psi_{s}\left(t^{*}\right) \geq N\right\}
$$


It follows from (3.1) that

$$
\begin{aligned}
& E\left(\chi_{\Omega_{N}}\left\|\Theta_{n}\left(t^{*}\right)\right\|_{\mathcal{H}}^{2}\right)=E\left(\chi_{\Omega_{N}}\left\|\Theta_{n}\left(t^{*} \wedge T_{N}\right)\right\|_{\mathcal{H}}^{2}\right) \\
& \quad \leq e^{N} E\left(\left\|h_{n}-h\right\|_{\mathcal{H}}^{2}\right)
\end{aligned}
$$

Now choose any $\delta>0$. By means of (3.4),

$$
\begin{aligned}
& P\left\{\left\|\Theta_{n}\left(t^{*}\right)\right\|_{\mathcal{H}}>\delta\right\} \\
& \leq P\left\{\Omega \backslash \Omega_{N}\right\}+P\left\{\chi_{\Omega_{N}}\left\|\Theta_{n}\left(t^{*}\right)\right\|_{\mathcal{H}}>\delta\right\} \\
& \quad \leq P\left\{\Psi_{s}\left(t^{*}\right) \geq N\right\}+\frac{e^{N}}{\delta^{2}} E\left(\left\|h_{n}-h\right\|_{\mathcal{H}}^{2}\right)
\end{aligned}
$$

For given $\epsilon>0$, choose $N$ in (3.2) so that

$$
P\left\{\Psi_{s}\left(t^{*}\right) \geq N\right\}<\epsilon
$$

Then, there is a positive integer $L=L(N, \delta)$ such that for all $n \geq L$,

$$
\frac{e^{N}}{\delta^{2}} E\left(\left\|h_{n}-h\right\|_{\mathcal{H}}^{2}\right)<\epsilon
$$

Hence,

$$
\left\|\Theta_{n}\left(t^{*}\right)\right\|_{\mathcal{H}} \rightarrow 0 \quad \text { in } P \text {-measure }
$$

In the same setting as above, we use Lebesgue's dominated convergence theorem to prove the following fact.

Lemma 3.2. Let $0 \leq s<t^{*}<\infty$. Choose any $Q \in \mathcal{F}_{s}$, and a bounded continuous function $F$ on $\mathcal{H}$. Then,

$$
E\left(\chi_{Q} F\left(Y\left(t^{*}, s ; h_{n}\right)\right)\right) \rightarrow E\left(\chi_{Q} F\left(Y\left(t^{*}, s ; h\right)\right)\right)
$$

Proof. Assume that the claim is false. Then, there is $\epsilon>0$ and a subsequence $\left\{h_{n_{k}}\right\}_{k=1}^{\infty}$ such that

$$
E\left(\chi_{Q}\left|F\left(Y\left(t^{*}, s ; h_{n_{k}}\right)\right)-F\left(Y\left(t^{*}, s ; h\right)\right)\right|\right)>\epsilon, \quad \forall k
$$

By Lemma 3.1, $\left\|Y\left(t^{*}, s ; h_{n_{k}}\right)-Y\left(t^{*}, s ; h\right)\right\|_{\mathcal{H}} \rightarrow 0$ in $P$-measure as $k \rightarrow \infty$. Thus, we can further extract a subsequence still denoted by $\left\{h_{n_{k}}\right\}_{k=1}^{\infty}$ such that

$$
\left\|Y\left(t^{*}, s ; h_{n_{k}}\right)-Y\left(t^{*}, s ; h\right)\right\|_{\mathcal{H}} \rightarrow 0, \quad P \text {-almost surely }
$$

It follows that

$$
\lim _{k \rightarrow \infty} E\left(\chi_{Q}\left|F\left(Y\left(t^{*}, s ; h_{n_{k}}\right)\right)-F\left(Y\left(t^{*}, s ; h\right)\right)\right|\right)=0
$$

which is a contradiction. 
It follows from Lemma 3.2 that $E\left(F\left(Y\left(t^{*}, s ; h\right)\right)\right)$ is continuous in $h \in \mathcal{H}$, which is the Feller property. Again by virtue of Lemma 3.2, we can repeat the same argument as in [4] or [18] to conclude that $Y(\cdot, \cdot ; \cdot)$ is an $\mathcal{H}$-valued Markov process.

We also need the following fact.

Lemma 3.3. Let $h \in \mathcal{H}$. For each $Q \in \mathcal{B}(\mathcal{H}), P\{Y(t, 0 ; h) \in Q\}$ is $\mathcal{B}([0, \infty))$ measurable in $t \geq 0$, and a probability measure $\mu_{T}$ on $\mathcal{H}$ can be defined by

$$
\mu_{T}(Q)=\frac{1}{T} \int_{0}^{T} P\{Y(t, 0 ; h) \in Q\} d t, \quad T>0
$$

Proof. For each bounded continuous function $F$ on $\mathcal{H}$,

$$
E(F(Y(t, 0 ; h)))=\int_{\mathcal{H}} F(y) P\{Y(t, 0 ; h) \in d y\}
$$

is continuous in $t \geq 0$. Thus, for each closed subset $Q$ of $\mathcal{H}$,

$$
P\{Y(t, 0 ; h) \in Q\}=\int_{\mathcal{H}} \chi_{Q}(y) P\{Y(t, 0 ; h) \in d y\}
$$

is upper semicontinuous in $t \geq 0$. By the Dynkin system theorem, $P\{Y(t, 0$; $h) \in Q\}$ is $\mathcal{B}([0, \infty))$-measurable for each $Q \in \mathcal{B}(\mathcal{H})$, and $\mu_{T}(Q)$ is well defined. By Lebesgue's dominated convergence theorem, it is easy to show that $\mu_{T}(\cdot)$ is countably additive and hence, a probability measure.

Choose any $u_{0} \in \mathcal{H}$, and let $u$ be the solution of (0.1)-(0.4) on the interval $[0, \infty)$. Let us fix a positive constant $K$, and set

$$
X(t)=\|u(t)\|_{\mathcal{H}}^{2}
$$

Then,

$$
\begin{aligned}
d X= & -2 \nu\|u(t)\|_{V}^{2} d t-2 J(u(t)) d t+2\langle f, u(t)\rangle_{V^{*}, V} d t \\
& +\sum_{k=1}^{\infty}\left\|h_{k}(u(t))\right\|_{\mathcal{H}}^{2} d t+\sum_{k=1}^{\infty} 2\left\langle u(t), h_{k}(u)\right\rangle_{\mathcal{H}} d B_{k}
\end{aligned}
$$

and thus, for $0<r<\frac{1}{2}$ and a nonnegative constant $\alpha$,

$$
\begin{aligned}
& E\left(e^{\alpha t}(K+X(t))^{r}\right)=E\left((K+X(0))^{r}\right) \\
& -2 \nu E\left(\int_{0}^{t} r e^{\alpha s}(K+X(s))^{r-1}\|u(s)\|_{V}^{2} d s\right) \\
& \quad-2 E\left(\int_{0}^{t} e^{\alpha s} r(K+X(s))^{r-1} J(u(s)) d s\right) \\
& \quad+2 E\left(\int_{0}^{t} e^{\alpha s} r(K+X(s))^{r-1}\langle f, u(s)\rangle_{V^{*}, V} d s\right)
\end{aligned}
$$




$$
\begin{aligned}
& +E\left(\int_{0}^{t} e^{\alpha s} \alpha(K+X(s))^{r} d s\right)+E\left(\sum_{k=1}^{\infty} \int_{0}^{t} r e^{\alpha s}(K+X(s))^{r-1}\left\|h_{k}(u(s))\right\|_{\mathcal{H}}^{2} d s\right) \\
& +E\left(\sum_{k=1}^{\infty} 2 \int_{0}^{t} r(r-1) e^{\alpha s}(K+X(s))^{r-2}\left|\left\langle u(s), h_{k}(u(s))\right\rangle_{\mathcal{H}}\right|^{2} d s\right)
\end{aligned}
$$

By (1.2),

$$
\begin{aligned}
& E\left(\sum_{k=1}^{\infty} \int_{0}^{t} r e^{\alpha s}(K+X(s))^{r-1}\left\|h_{k}(u(s))\right\|_{\mathcal{H}}^{2} d s\right) \\
& \leq C_{1} E\left(\int_{0}^{t} r e^{\alpha s}(K+X(s))^{r} d s\right)+C_{2} \int_{0}^{t} r e^{\alpha s} K^{r-1} d s
\end{aligned}
$$

For all $a \geq 0, b \geq 0$ and $0<\delta<1$,

$$
a^{2} \geq(a+b)^{2}(1-\delta)-C_{\delta} b^{2}
$$

holds with $C_{\delta}=\frac{1}{\delta}-1$. Using this and (1.10),

$$
\begin{aligned}
-E & \left(\sum_{k=1}^{\infty} 2 \int_{0}^{t} r(1-r) e^{\alpha s}(K+X(s))^{r-2}\left|\left\langle u(s), h_{k}(u(s))\right\rangle_{\mathcal{H}}\right|^{2} d s\right) \\
\leq & -C_{4}(1-\delta) E\left(2 \int_{0}^{t} r(1-r) e^{\alpha s}(K+X(s))^{r} d s\right) \\
& +2 \int_{0}^{t} r(1-r) e^{\alpha s}\left(C_{\delta} K^{r}+C_{5} K^{r-2}\right) d s
\end{aligned}
$$

By (1.11), we can choose $0<r<\frac{1}{2}$ and $0<\delta<1$ such that

$$
C_{1}<C_{4}(1-\delta) 2(1-r)
$$

We also note that

$$
\begin{aligned}
E\left(\int_{0}^{t} e^{\alpha s} \alpha(K+X(s))^{r} d s\right) \leq & E\left(\int_{0}^{t} e^{\alpha s} \alpha(K+X(s))^{r-1} X(s) d s\right) \\
& +\alpha \int_{0}^{t} e^{\alpha s} K^{r} d s
\end{aligned}
$$

and

$$
\begin{aligned}
& E\left(\int_{0}^{t} e^{\alpha s} r(K+X(s))^{r-1}\left|\langle f, u(s)\rangle_{V^{*}, V}\right| d s\right) \\
& \quad \leq \frac{\nu}{4} E\left(\int_{0}^{t} r e^{\alpha s}(K+X(s))^{r-1}\|u(s)\|_{V}^{2} d s\right)+\frac{\|f\|_{V^{*}}^{2}}{\nu} \int_{0}^{t} e^{\alpha s} r K^{r-1} d s
\end{aligned}
$$

It follows that

$$
\begin{aligned}
& E\left(e^{\alpha t}(K+X(t))^{r}\right)+\nu E\left(\int_{0}^{t} r e^{\alpha s}(K+X(s))^{r-1}\|u(s)\|_{V}^{2} d s\right) \\
& \leq E\left((K+X(0))^{r}\right)+\left(C(r, K)+\alpha K^{r}\right) \int_{0}^{t} e^{\alpha s} d s, \quad \forall t>0
\end{aligned}
$$


for every $\alpha \geq 0$ satisfying

$$
\alpha\|v\|_{\mathcal{H}}^{2}<\frac{\nu}{2} r\|v\|_{V}^{2}, \quad \forall v \in V
$$

By choosing $\alpha>0$ satisfying (3.9), we derive from (3.8) that

$$
E\left((K+X(t))^{r}\right) \leq C, \quad \forall t>0
$$

for some constant $C>0$.

Next by choosing $\alpha=0$ in (3.8), we have

$$
\frac{1}{T} \int_{0}^{T} E\left(r(K+X(t))^{r-1}\|u(t)\|_{V}^{2}\right) d t \leq C
$$

for all $T \geq 1$, for some positive constant $C$.

Let us write

$$
\begin{aligned}
& \mathcal{A}_{L}(t)=\left\{\omega \mid(K+X(t))^{r} \geq L\right\} \\
& \mathcal{B}_{M}(t)=\left\{\omega \mid r(K+X(t))^{r-1}\|u(t)\|_{V}^{2} \geq M\right\} \\
& \mathcal{C}_{N}(t)=\left\{\omega \mid\|u(t)\|_{V}^{2} \geq N\right\}
\end{aligned}
$$

Then,

$$
\frac{1}{T} \int_{0}^{T} P\left\{\mathcal{A}_{L}(t)\right\} d t \leq \frac{C}{L}
$$

and

$$
P\left\{\mathcal{C}_{N}(t)\right\} \leq P\left\{\mathcal{A}_{L}(t)\right\}+P\left\{\mathcal{C}_{N}(t) \backslash \mathcal{A}_{L}(t)\right\}
$$

But if $M=r N L^{1-\frac{1}{r}}$,

$$
\mathcal{C}_{N}(t) \backslash \mathcal{A}_{L}(t) \subset \mathcal{B}_{M}(t)
$$

and hence, by (3.11),

$$
\frac{1}{T} \int_{0}^{T} P\left\{\mathcal{C}_{N}(t) \backslash \mathcal{A}_{L}(t)\right\} d t \leq \frac{C L^{\frac{1}{r}-1}}{r N}, \quad \forall T \geq 1
$$

It follows that

$$
\begin{aligned}
& \frac{1}{T} \int_{0}^{T} P\left\{\mathcal{C}_{N}(t)\right\} d t \leq \frac{1}{T} \int_{0}^{T} P\left\{\mathcal{A}_{L}(t)\right\} d t \\
& \quad+\frac{1}{T} \int_{0}^{T} P\left\{\mathcal{C}_{N}(t) \backslash \mathcal{A}_{L}(t)\right\} d t \\
& \leq \frac{C}{L}+\frac{C L^{\frac{1}{r}-1}}{r N}, \quad \forall T \geq 1
\end{aligned}
$$

Now for given $\epsilon>0$, we can choose large $L>0$ so that

$$
\frac{C}{L}<\epsilon
$$


and then, choose large $N$ so that

$$
\frac{C L^{\frac{1}{r}-1}}{r N}<\epsilon
$$

It follows that

$$
\frac{1}{T} \int_{0}^{T} P\left\{\|u(t)\|_{V}^{2} \leq N\right\} d t>1-2 \epsilon, \quad \forall T \geq 1
$$

Since each closed ball in $V$ of finite radius is a compact subset of $\mathcal{H},(3.12)$ implies that the family $\left\{\mu_{T}\right\}_{T \geq 1}$ of probability measures defined in Lemma 3.3 is tight. Now the existence of an invariant measure follows by the method of Krylov-Bogoliubov.

\section{Proof of Theorem 1.6}

Under the conditions (1.3), (1.12)-(1.14) and (1.17), let $u$ be the solution of $(0.1)-(0.4)$. As mentioned above, the unique solution exists on the interval $[0, \infty), P$-almost surely. We write

$$
X(t)=\|u(t)\|_{\mathcal{H}}^{2}
$$

By Ito's formula, we have for each $\eta>0, \lambda>0$, and $0<r<\frac{1}{2}$,

$$
\begin{aligned}
& E\left(e^{\lambda t}(\eta+X(t))^{r}\right)=E\left((\eta+X(0))^{r}\right) \\
& \quad-2 r \nu E\left(\int_{0}^{t} e^{\lambda s}(\eta+X(s))^{r-1}\|u(s)\|_{V}^{2} d s\right) \\
& \quad-2 r E\left(\int_{0}^{t} e^{\lambda s}(\eta+X(s))^{r-1} J(u(s)) d s\right) \\
& \quad+2 r E\left(\int_{0}^{t} e^{\lambda s}(\eta+X(s))^{r-1}\langle u(s), f(s)\rangle_{\mathcal{H}} d s\right) \\
& \quad+\lambda E\left(\int_{0}^{t} e^{\lambda s}(\eta+X(s))^{r} d s\right)+r E\left(\sum_{k=1}^{\infty} \int_{0}^{t} e^{\lambda s}(\eta+X(s))^{r-1}\left\|h_{k}(u(s))\right\|_{\mathcal{H}}^{2} d s\right) \\
& \quad-2 r(1-r) E\left(\sum_{k=1}^{\infty} \int_{0}^{t} e^{\lambda s}(\eta+X(s))^{r-2}\left|\left\langle u(s), h_{k}(u(s))\right\rangle_{\mathcal{H}}\right|^{2} d s\right), \quad \forall t \geq 0
\end{aligned}
$$

By (1.17), we can choose a number $K_{3}$ such that

$$
0<K_{3} \leq \frac{2 g}{K_{1}}-\|f\|_{L^{\infty}(0, \infty ; \mathcal{H})}
$$

We set

$$
K_{4}=2(1-r) C_{4}-C_{1}
$$

and choose $\lambda$ such that

$$
0<\lambda<\frac{2 r \nu}{K_{2}^{2}}+r K_{4}
$$


where $K_{2}$ is the constant in (1.16). Let $\tau$ be the stopping time defined by (1.18). Then, by Fatou's lemma and (1.15),

$$
\begin{aligned}
& \left(\frac{2 g}{K_{1}}-\|f\|_{L^{\infty}(0, \infty ; \mathcal{H})}\right) E\left(\int_{0}^{\tau \wedge t} e^{\lambda s} X(s)^{r-\frac{1}{2}} d s\right) \\
& \quad \leq E\left(\int_{0}^{\tau \wedge t} e^{\lambda s} \underline{\lim _{\eta \rightarrow 0}}(\eta+X(s))^{r-1}\left[J(u(s))-\langle u(s), f(s)\rangle_{\mathcal{H}}\right] d s\right) \\
& \quad \leq \varliminf_{\eta \rightarrow 0} E\left(\int_{0}^{\tau \wedge t} e^{\lambda s}(\eta+X(s))^{r-1}\left[J(u(s))-\langle u(s), f(s)\rangle_{\mathcal{H}}\right] d s\right), \quad \forall t \geq 0
\end{aligned}
$$

Now we note that (4.1) is still valid with $t$ replaced by any bounded stopping time. By combining (1.12), (1.16) and (4.2)-(4.5), we pass $\eta \rightarrow 0$ in (4.1) to obtain

$$
\begin{aligned}
& E\left(e^{\lambda(\tau \wedge t)} X(\tau \wedge t)^{r}\right)+2 r K_{3} E\left(\int_{0}^{\tau \wedge t} e^{\lambda s} X(s)^{r-\frac{1}{2}} d s\right) \\
& \quad+\left(\frac{2 r \nu}{K_{2}^{2}}+r K_{4}-\lambda\right) E\left(\int_{0}^{\tau \wedge t} e^{\lambda s} X(s)^{r} d s\right) \leq E\left(X(0)^{r}\right), \quad \forall t \geq 0
\end{aligned}
$$

Let us choose a number $p$ such that

$$
\frac{1}{p}=\frac{1}{r}+\frac{2}{1-2 r}
$$

Then, $0<p<\min \left(r, \frac{1}{2}-r\right)$, and by virtue of (4.6)-(4.7) and the inequality

$$
a^{\eta} b^{1-\eta} \leq \eta a+(1-\eta) b, \quad \forall a \geq 0, \quad \forall b \geq 0, \quad 0<\forall \eta<1
$$

we find that

$$
\begin{aligned}
& E\left(\int_{0}^{\tau \wedge t} e^{\lambda s} d s\right)=E\left(\int_{0}^{\tau \wedge t} e^{\lambda s} X(s)^{-p} X(s)^{p} d s\right) \\
& \quad \leq C E\left(\int_{0}^{\tau \wedge t} e^{\lambda s} X(s)^{r-\frac{1}{2}} d s\right)+E\left(\int_{0}^{\tau \wedge t} e^{\lambda s} X(s)^{r} d s\right) \leq C E\left(X(0)^{r}\right), \quad \forall t \geq 0 .
\end{aligned}
$$

where $C$ denotes positive constants independent of $u_{0}$ and $t$. Hence, we find that

$$
E\left(e^{\lambda \tau}-1\right) \leq \varliminf_{t \rightarrow \infty} E\left(\lambda \int_{0}^{\tau \wedge t} e^{\lambda s} d s\right) \leq C E\left(X(0)^{r}\right)
$$

for some constant $C>0$ independent of $u_{0}$. Thus, for each $t>0$,

$$
\left(e^{\lambda t}-1\right) P\{\tau \geq t\} \leq C E\left(X(0)^{r}\right)
$$

which proves (1.20).

We proceed to prove (1.19). Recalling (1.12), let us set

$$
Z(t)=e^{-C_{1} t}\|u(t)\|_{\mathcal{H}}^{2}
$$


Then, by (1.12) and (1.17), it holds $P$-almost surely that

$$
Z(t) \leq Z(s)+2 \sum_{k=1}^{\infty} \int_{s}^{t} e^{-C_{1} \eta}\left\langle h_{k}(u(\eta)), u(\eta)\right\rangle_{\mathcal{H}} d B_{k}(\eta), \quad \forall t \geq s \geq 0
$$

Therefore, the stochastic process $Z(t)$ is a nonnegative continuous supermartingale. Choose any positive integer $N$ and set

$$
\Omega_{N}=\{\omega \mid \tau(\omega) \leq N\}
$$

Then, $\Omega_{N} \in \mathcal{F}_{\tau \wedge N}$, and

$$
\int_{\Omega_{N}} Z(\tau \wedge N) d P=\int_{\Omega_{N}} Z(\tau) d P=0
$$

By the Optional Sampling theorem (see [13]),

$$
\int_{\Omega_{N}} Z((\tau+r) \wedge(N+r)) d P \leq \int_{\Omega_{N}} Z(\tau \wedge N) d P=0
$$

for every positive rational number $r$. Since $Z(t)$ is continuous in $t, P$-almost surely, (4.8) implies that there is $\tilde{\Omega}_{N} \subset \Omega_{N}$ such that

$P\left(\Omega_{N} \backslash \tilde{\Omega}_{N}\right)=0$, and, for each $\omega \in \tilde{\Omega}_{N}$,

$$
Z(\omega, t)=0, \quad \forall t>\tau(\omega)
$$

It follows from $(1.20)$ that $\tau<\infty, P$-almost surely. Consequently, there is $\tilde{\Omega}$ such that $P(\Omega \backslash \tilde{\Omega})=0$, and, for each $\omega \in \tilde{\Omega}$,

$$
Z(\omega, t)=0, \quad \forall t>\tau(\omega)
$$

This yields (1.19), and the proof of Theorem 1.6 is complete.

\section{References}

[1] Barbu, V., Da Prato, G., Röckner, M.: Stochastic porous media equations and self-organized criticality. Commun. Math. Phys. 285, 901-923 (2009)

[2] Barbu, V., Da Prato, G., Röckner, M.: Finite time extinction for solutions to fast diffusion stochastic porous media equations. C. R. Math. Acad. Sci. Paris 347, 81-84 (2009)

[3] Chow, P.L., Khasminskii, R.Z.: Stationary solutions of nonlinear stochastic evolution equations. Stoch. Anal. Appl. 15, 671-699 (1997)

[4] Da Prato, G., Zabczyk, J.: Stochastic Equations in Infinite Dimensions. Cambridge University Press, Cambridge (1992)

[5] Diaz, J.I.: Special finite time extinction in nonlinear evolution systems: dynamic boundary conditions and Coulomb friction type problems. Prog Nonlinear Differ. Equ. 64, 71-97 (2005) 
[6] Diaz, J.I., Glowinski, R., Guidoboni, G., Kim, T.: Qualitative properties and approximation of solutions of Bingham fluids: on the stabilization for large time and the geometry of the support. Rev. R. Acad. Cienc. Exactas Fis. Nat. Ser A Mat. 104, 153-196 (2010)

[7] Diestel, J., Uhl, J.J.: Vector Measures. American Mathematical Society, Providence (1977)

[8] Duvaut, G., Lions, J.L.: Inequalities in Mechanics and Physics. Springer, Berlin (1976)

[9] Flandoli, F.: Dissipativity and invariant measures for stochastic Navier-Stokes equations. NoDEA 1, 403-423 (1994)

[10] Flandoli, F.: An introduction to 3D stochastic fluid dynamics. In: Lecture Notes in Mathematics, vol. 1942. CIME Lecture Series (Summer 2005). Springer, Berlin (2008)

[11] Flandoli, F., Gatarek, D.: Martingale and stationary solutions for stochastic Navier-Stokes equations. Probab. Theory Relat. Fields 102, 367-391 (1995)

[12] Glowinski, R.: Numerical Methods for Nonlinear Variational Problems. Springer, Berlin (1984)

[13] Haussmann, U.G., Pardoux, E.: Stochastic variational inequalities of parabolic type. Appl. Math. Optim. 20, 163-192 (1989)

[14] Karatzas, I., Shreve, S.: Brownian Motion and Stochastic Calculus, 2nd edn. Springer, New York (1997)

[15] Krylov, N.V., Rozovskii, B.L.: Stochastic evolution equations. In: Current Problems in Mathematics, vol. 14, pp. 71-147, 256 (1979) (Akad. Nauk SSSR, Vsesoyuz. Inst. Nauchn. i Tekhn. Informatsii, Moscow)

[16] Liu, W., Röckner, M.: SPDE in Hilbert space with locally monotone coefficients. J. Funct. Anal. 259, 2902-2922 (2010)

[17] Menaldi, J.L., Sritharan, S.S.: Stochastic 2-D Navier-Stokes equations. Appl. Math. Optim. 46, 31-53 (2002)

[18] Prévôt, C., Röckner, M.: A concise course on stochastic partial differential equations. In: Lecture Notes in Mathematics, vol. 1905. Springer, Berlin (2007)

[19] Strauss, M.J.: Variations of Korn's inequality and Sobolev's inequalities, partial differential equations. In: Proceedings of Symposia in Pure Mathematics, vol. 23, pp. 207-214. AMS, Providence (1971) 
Jong Uhn Kim

Department of Mathematics

Virginia Tech

Blacksburg

VA 24061-0123

USA

e-mail: kim@math.vt.edu

Received: 17 April 2012.

Accepted: 5 August 2012. 